\title{
Leadless pacing in the elderly: never too old for something new
}

\author{
Valentina Barletta, Giulio Zucchelli, Matteo Parollo, Mario Giannotti Santoro, Silvio Tolve, Andrea Di Cori, \\ Luca Segreti, Raffaele De Lucia, Maria Grazia Bongiorni
}

Second Department of Cardiology, Cardiac Thoracic and Vascular Department, University Hospital of Pisa, Italy

\begin{abstract}
Rates of cardiac pacemaker implantation rise with age, and, meanwhile, elderly patient may be at great risk of complications, as pneumothorax, lead perforation, or pocket dehiscence. The use of leadless pacemaker could overcome peri- and post-procedural complications related to the presence of transvenous leads and pocket. The study aims to investigate feasibility and outcomes of Micra Transcatheter Pacing System (M-TPS) implantation in elderly, which represents a challenge for conventional cardiac pacing. Between May 2014 and July 2019, 109 patients (88 males, mean age $77.71 \pm 9.68$ years) underwent M-TPS implantation at our Center, targeting a non-apical site of delivery when feasible. Study population was divided into two groups according to age (group 1 $<79$ years $v s$ group 2 group $2{ }^{3} 80$ years). The outcome evaluation included electrical performance at hospital discharge, and during follow-up. In 46/109 cases (34 males, 73.91\%) M-TPS was
\end{abstract}

Correspondence: Valentina Barletta, Azienda Ospedaliero - Universitaria Pisana, Via Paradisa 2, 56100 Pisa, Italy.

Tel. +39.050.993043 - Fax: +39.050.992351.

E-mail: valentinabarletta@hotmail.com

Key words: Leadless pacemaker; lead extraction; Micra; cardiac pacing; elderly.

Conflict of interest: MGB received honoraria or consultation fees from Medtronic, Boston Scientific, and Abbott. All the other authors have no conflict of interest to declare.

Contributions: All the authors contributed to the design and implementation of the research, to the analysis of the results and to the writing of the manuscript. All the authors have read and approved the final version of the manuscript and agreed to be accountable for all aspects of the work.

Ethics approval and consent to participate: The study was conformed to the Declaration of Helsinki on human research. Written informed consent was obtained from every patient.

Received for publication: 23 February 2020.

Accepted for publication: 16 April 2020.

${ }^{\circ}$ Copyright: the Author(s), 2020

Licensee PAGEPress, Italy

Monaldi Archives for Chest Disease 2020; 90:1255

doi: 10.4081/monaldi.2020.1255

This article is distributed under the terms of the Creative Commons Attribution Noncommercial License (by-nc 4.0) which permits any noncommercial use, distribution, and reproduction in any medium, provided the original author(s) and source are credited. implanted in patients older than 80 years. There were no statistically significant differences between groups for demographics characteristics, except for age. The procedure was performed via the right femoral access in 102/109 cases and was successful in all cases, with no device-related events. No differences were observed between groups in procedure duration, single device delivery, electrical performance at implant and at 12 month F-U. MTP-S implant is an effective and safe procedure in elderly patients, with similar electrical performance and outcome compared with younger patients at mid-term follow-up.

\section{Introduction}

The development of electronic heart control is one of the greatest triumphs achieved by modern technology. Two factors were decisive in obtaining the remarkable technological progress in the field of cardiac pacing: the first is the great progress in electronics during the first half of the $20^{\text {th }}$ century, and the second is the close collaboration between doctors and engineers.

The extensive use of cardiac implantable electronic devices (CIED) has undoubtedly improved both the prognosis and the quality of life of patients suffering from arrhythmias [1], and the number of CIED has been increasing year-on-year [2]. This, with improvements in life expectancy, means that more elderly patients will meet the criteria for cardiac pacing. Indeed, over $80 \%$ of pacemakers are implanted in patients over 80 years [3], who also have more comorbidities than the general population. Nevertheless, device implantation is associated with significant complications, mainly related to intravenous leads and subcutaneous pockets created for the generator [4].

The study aims at investigating feasibility and clinical and electrical outcomes of Micra Transcatheter Pacing System (MTPS) implantation in a specific patient population, like the elderly, which represent a challenge for conventional cardiac pacing.

\section{Materials and Methods}

\section{Patient population}

Between May 2014 and July 2019, 109 patients (88 males, $76.15 \%$, mean age $77.71 \pm 9.68$ ) underwent M-TPS implantation in our Center (UOC Cardiologia 2 - Azienda OspedalieroUniversitaria Pisana, Pisa, Italy), targeting a non-apical site of delivery when feasible. All patients fulfilled standard criteria for pacemaker implantation with specific indication to receive VVI pacing. The exclusion criteria for Micra implant were age $<18$ years, hemodynamic instability, mechanical tricuspid valve prosthesis or inferior vena cava filter, morbid obesity that could impair 
remote Micra control, femoral venous occlusion, allergy to MicraTPS components. The study was conformed to the Declaration of Helsinki on human research. Written informed consent was obtained from every patient.

Study population was divided into two groups according to age (group $1<79$ years $v s$ group $2{ }^{3} 80$ years).

\section{Implant procedure}

The implantation procedure of the M-TPS device has been already largely described in detail [5]. Briefly, the device is implanted using a 23-F internal diameter/27-F outer diameter introducer through a femoral vein and the inferior vena cava towards the right ventricle using a steerable catheter. The M-TPS is then anchored to the myocardium via 4 electrically inactive, flexible, nitinol tines. After verifying device fixation, confirmed by the movement of at least two of four tines, and obtaining adequate electrical measurement (namely pacing threshold $<1.5 \mathrm{~V}$ at pulse duration of $0.24 \mathrm{~ms}$ ), a tether is cut and the delivery system is removed, otherwise repositioning to another site of the right ventricle can be attempted. Usually, the figure of eight stitch is used to guarantee a correct hemostasis at the access site and removed after 24 hours. See Figure 1 A,B for a representation of device final deployment position.

\section{Follow-up}

Procedural (implant duration, number of deployments, and adverse events) and device data (pacing capture threshold, R-wave amplitude, and pacing impedance) were prospectively collected.
All patients underwent electrocardiography and chest radiography before hospital discharge. Every subject was evaluated for adverse events and device function at hospital discharge and during followup. Follow-up was planned at 1, 6, and 12 months and then annually. Specifically, at every time point the automated measurements of the device were reviewed, and the adequacy of the programming and pacing parameters confirmed. High pacing threshold (HPT) was defined as $>1.0 \mathrm{~V}$ at pulse duration of $0.24 \mathrm{~ms}$.

\section{Statistical analysis}

Statistical analysis was performed with NCSS 12 Statistical Software, version 2018 (NCSS, LLC, Kaysvill, UT, USA). Categorical data are expressed as percentages, and continuous variables as mean \pm standard deviation or median [interquartile range (IQR)], as appropriate. Parametric continuous variables were evaluated using unpaired and paired $t$-tests. Non-parametric continuous variables were evaluated using the Mann-Whitney $U$ test. The Fisher's exact test was used to compare categorical variables. All tests were two-sided; a $\mathrm{p}<0.05$ was considered to be significant.

\section{Results}

\section{Characteristics of the study population and acute outcomes}

The demographics and clinical data of the entire patients' population and each group are reported in Table 1. In 46/109 cases (34

Table 1. Demographic characteristics.

\begin{tabular}{|c|c|c|c|c|}
\hline Demographics & Overall & Elderly & Non elderly & p \\
\hline Males, n (\%) & $83(76.15)$ & $34(73.91)$ & $49(77.78)$ & ns \\
\hline Age (years) & $77.71 \pm 9.68$ & $85.85 \pm 4.22$ & $71.57 \pm 7.95$ & $<0.001$ \\
\hline LVEF (\%) & $56.00 \pm 7.80$ & $55.43 \pm 8.04$ & $57.00 \pm 7.63$ & ns \\
\hline
\end{tabular}

ns, not significant; LVEF, left ventricular ejection fraction.

A

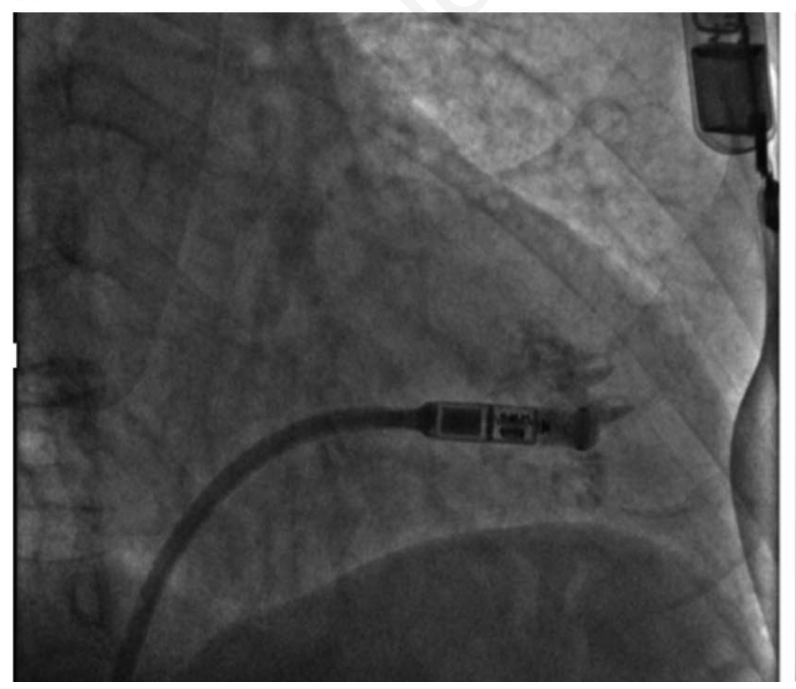

B

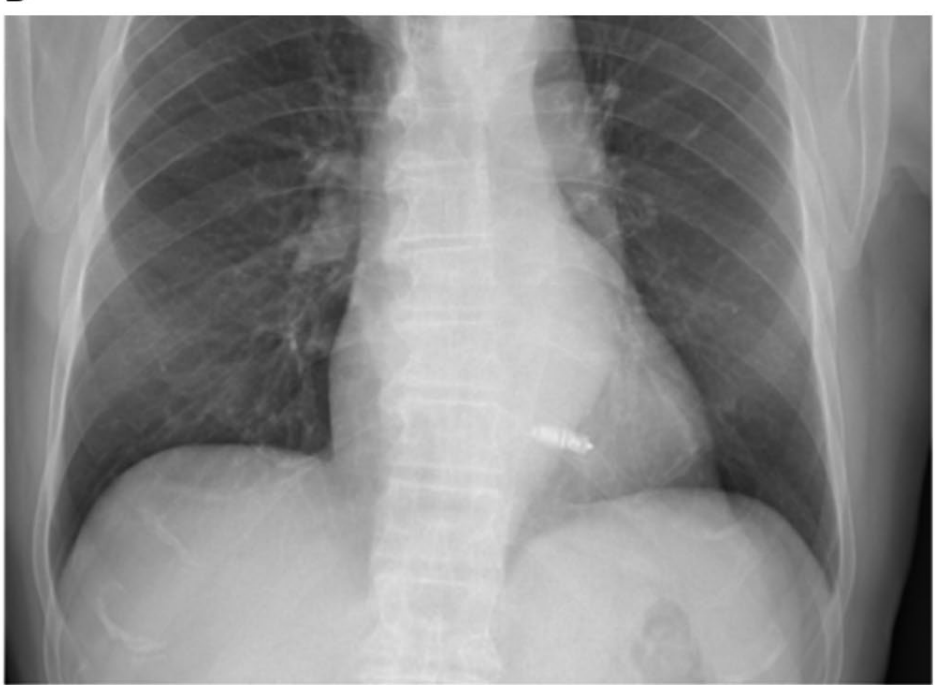

Figure 1. A) Micra-TPS deployment (fluoroscopic frame in OAS). B) Micra-TPS final position, chest X ray (frontal frame). 
males, 73.91\%) M-TPS was implanted in patients older than 80 years. The primary indications for M-TPS implant were bradycardia associated with persistent or permanent atrial fibrillation $(51.00 \%)$, sinus-node dysfunction $(16.00 \%)$, and paroxysmal atrioventricular blocks $(22.00 \%)$. The predominant reasons for the selection of VVI pacing were atrial tachyarrhthmia (25.51\%), an expected low pacing frequency $(11.22 \%)$, advanced age/frailty $(12.24 \%)$, or patients' preference $(10.20 \%)$. There were no statistically significant differences between groups for demographics characteristics, except for age. Complete procedural success was achieved in all cases and in the majority of procedures (102, $94.4 \%$ ) the right femoral access was used.

The major technical details of implant procedure are reported in Table 2, and device parameters at implant and follow-up visits are showed in Figure 2. No differences were observed between groups in procedure features as duration, single device delivery (group 1 vs group 2: $57.38 \%$ vs $69.05 \%, \mathrm{p}=0.27$ ), fluoroscopy time (group $1 v s$ group 2: $12.98 \pm 8.24 v s 13.53 \pm 8.43 \mathrm{~min}, \mathrm{p}=0.65$ ), electrical performance at implant (group $1 v s$ group 2: pacing threshold $0.67 \pm 0.39 \mathrm{~V} / 0.24 \mathrm{~ms} v s 0.57 \pm 0.33 \mathrm{~V} / 0.24 \mathrm{~ms}, \mathrm{p}=0.70$; impedance $714.03 \pm 69.91 \mathrm{Ohm} v s 723.78 \pm 28 \mathrm{Ohm}, \mathrm{p}=0.99$; R wave amplitude $9.88 \pm 4.42 \mathrm{mV}$ vs $9.73 \pm 4.68 \mathrm{mV}, \mathrm{p}=0.68$ ). The implant site was usually the septum in both groups (group 1 vs group 2: $86.89 \%$ vs $64.44 \%, \mathrm{p}=0.006)$.

\section{Electrical parameters during follow-up}

On implantation, the average pacing threshold was $0.57 \pm 0.36$ $\mathrm{V} / 0.24 \mathrm{~ms}$. The mean R-wave amplitude was $9.82 \pm 4.51 \mathrm{mV}$, and the average pacing impedance was $718.13 \pm 176.38 \mathrm{Ohm}$. As shown in Figure 2, in the entire population the major electrical variables stabilized during follow-up and remained stable; no significant differences were observed between groups at 12 month F-U (group 1 vs group 2: pacing threshold $0.59 \pm 0.37 \mathrm{~V} / 0.24 \mathrm{~ms}$ vs $0.54 \pm 0.24$ $\mathrm{V} / 0.24 \mathrm{~ms}, \mathrm{p}=0.85$; impedance $575.52 \pm 115.06$ Ohm $v s$ $599.09 \pm 86.13 \mathrm{Ohm}, \mathrm{p}=0.42$; R wave amplitude $12.62 \pm 5.18 \mathrm{mV} v \mathrm{~s}$ $11.62 \pm 5.04 \mathrm{mV}, \mathrm{p}=0.51)$.

\section{Clinical follow-up}

The patients were followed-up for an average of 18.05 months (median 12 months, maximum follow 60 months for 5 patients, 24 patients reached the 48-months follow-up). No acute complications were reported and, in particular, we did not observe neither venous access issues, nor fever or acute signs of infection after MTPS implant. No device-related events were registered during follow-up and, in particular, no device infection and/or malfunction were reported.

\section{Discussion}

The main finding of this study is the confirmation of safety and effectiveness of Micra-TPS implant even in older patients, with electrical performance and outcomes comparable with the general population at follow-up. This appealing opportunity seems particularly interesting in the contemporary clinical scenario.

\section{Leadless pacing: the starting point}

The extensive use of cardiac implantable electronic devices (CIED) has undoubtedly improved both the prognosis and the quality of life of patients suffering from arrhythmias [1], and the number of CIED has been increasing year-on-year [2]. This, with improvements in life expectancy, means that more elderly patients will meet the criteria for cardiac pacing. Indeed, over $80 \%$ of pacemakers are implanted in patients over 80 years [3], who also have more comorbidities than the general population. Nevertheless, device implantation is associated with significant complications, mainly related to intravenous leads and subcutaneous pockets created for the generator [4]. Adverse events related to the use of CIED can arise at any time in the "device history": acute complications are essentially related to the surgical treatment, whilst long-term complications are more frequently caused by infections affecting the device, or its malfunction, occurring especially after re-operation for generator replacement or upgrading, due to longer procedure times and reduced vascularization or local reactive fibrosis.

Leadless pacemakers represent one of the most important technological advances in the field of cardiac pacing. The first efforts to design implantable systems date back to the 1970s [6], but only recently technology has reached the market for clinical human applications as an alternative to traditional systems in order to reduce many of the aforementioned complications [5,7].

Currently, two self-contained right ventricular pacemakers implanted by using a femoral percutaneous approach have been developed to overcome these limits, although only one is still available for clinical purpose. Indeed, the Nanostim pacemaker (St. Jude Medical/Abbott Laboratories, Chicago, IL, USA) was removed from the market due to a premature battery depletion in implanted devices. Micra Transcatheter Pacing System (M-TPS; Medtronic, Minneapolis, MN, USA) is the unique device currently available worldwide for leadless pacing. It is composed of an introducer, a delivery system and the leadless pacemaker $\left(0.8 \mathrm{~cm}^{3}, 2.0 \mathrm{~g}\right)$.

The main limitation of leadless pacemakers is the current availability of a single chamber only stimulation: the primary indication, according to ESC guidelines [1], is a bradycardia associated with a persistent or permanent atrial tachyarrhythmia, and this indication has been confirmed in our real-life cohort. Other indications could lead to VVI pacemaker selection, such as an atrioventricular block or a sinus node dysfunction, if frequent pacing is not expected. All these observations allow to consider the leadless option even in patients who had previously undergone PM extraction and require to be re-implanted. Moreover, implementation of dedicated algorithms in Micra TPS allows for a VDD pacing with the single ventricular components [8,] expanding the field of application.

Table 2. Procedural details.

\begin{tabular}{lccc} 
Procedural & Overall & Elderly & Non elderly \\
Fluoroscopy time, min & $13.22 \pm 8.28$ & $13.53 \pm 8.43$ & $12.97 \pm 8.24$ \\
Single deployment, $\mathrm{n}(\%)$ & $64(62.14)$ & $29(69.05)$ & $35(57.38)$ \\
\hline Right femoral access, $\mathrm{n}(\%)$ & $102(94.4 \%)$ & $45(97.83 \%)$ & $\mathrm{ns}$ \\
\hline
\end{tabular}

ns, not significant. 


\section{A. Mean Threshold}
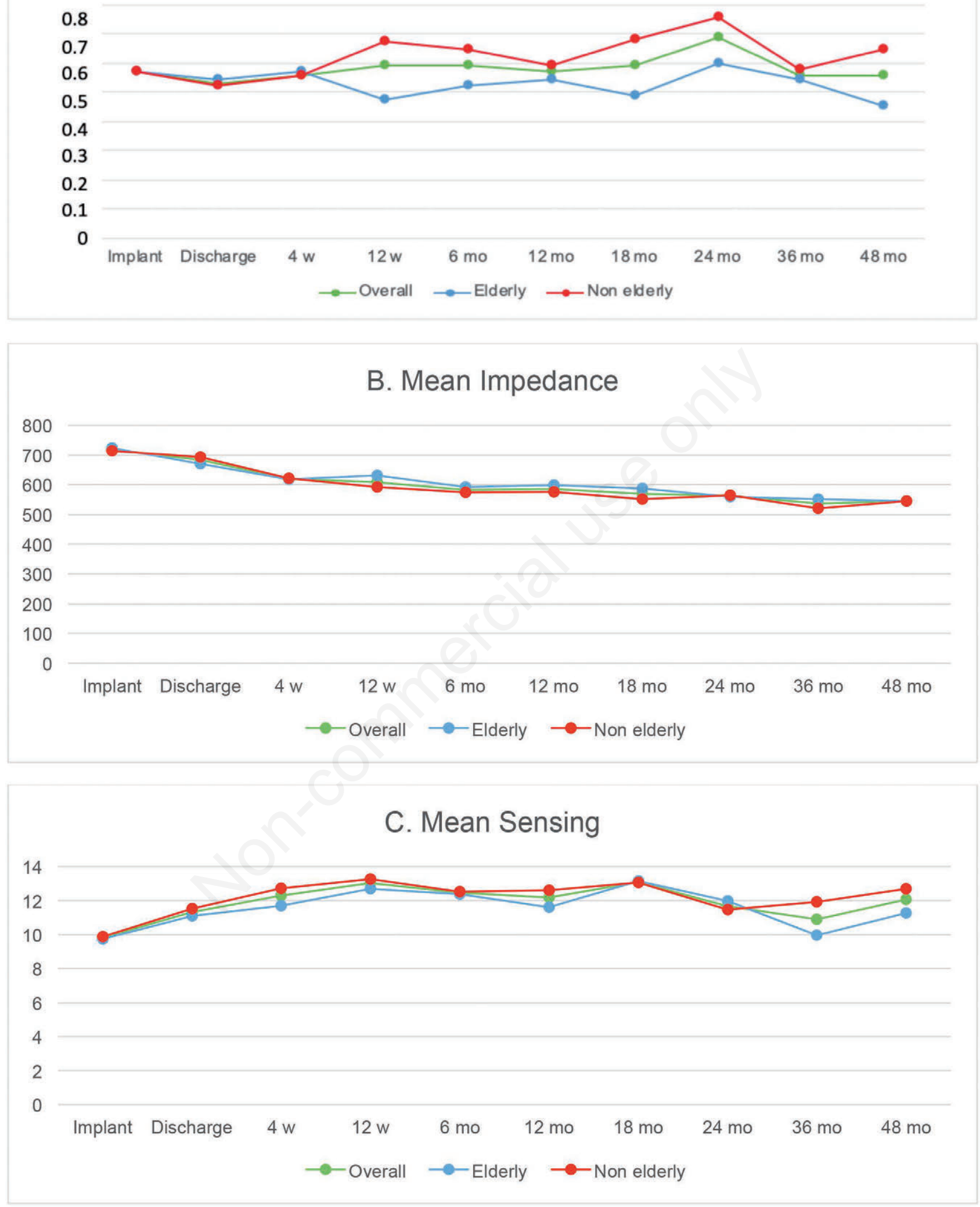

Figure 2. Electrical performance: from implant throughout follow-up. Pacing thresholds were achieved at pulse duration of 0.24 ms. A) Mean threshold values expressed in $\mathrm{mV}$ and achieved at pulse duration of $0.24 \mathrm{~ms}$; green: overall patients' population; red: Group 1 ; blue: Group 2.. B) Mean impedance values expressed in Ohm; green: overall patients' population; red: Group 1; blue: Group 2. C) Mean pacing sensing values expressed in mV; green: overall patients' population; red: Group 1; blue: Group 2. 


\section{Leadless pacing: safety first}

In recent years, safety and effectiveness of Micra TPS have been largely demonstrated [9] and our results are a further confirmation of this positive trend. The early performance of the device was published in 2015 by Ritter et al. [10]: no serious adverse device events occurred and, considering the 726 patients enrolled in the study, implant procedure was successful in more than $99 \%$ of cases. Furthermore during the entire follow-up no cases of dislocation or system infection were observed, maintaining an excellent electrical performance [5]. Afterwards, in the Micra TPS post-approval registry, the observation was extended to more than 1800 patients of a real-world population, including patients with valve prosthesis or with pre-existing CIED [11]. Authors reported a very low incidence of major complications $(1.52 \%)$ with only $0.13 \%$ of cardiac perforation. Such favorable outcomes have also been confirmed in a comparison with traditional transvenous pacemakers [12]. Since the original Micra TPS trial the authors found a 51\% major complication decrease compared with a historical cohort of patients enrolled in previous studies. More recently, El-Chami et al. [12] compared the rate of complications between leadless pacemakers and single/dual chamber PM, concluding for a more favorable safety outcome with the leadless solution. More precisely, it's pivotal to point the attention to the reduction in the incidence rate of one of the most feared complications during a pacemaker implantation procedure, which is cardiac perforation. In detail, the rate of apical Micra-TPS positioning tended to decrease during time (53\% in the early trial group $v s$ $34 \%$ in the following cohort), suggesting that a non-apical device deployment can be safely achieved in the great majority of patients. In addition to safety, also electrical performance of M-TPS in a nonapical position (septal/right ventricle outflow tract) has already been reported [13], emphasizing the stability of the major electrical parameters at short-to-mid follow-up.

The demonstrated safety of Micra implant procedure has made this technique appealing also in ageing population, traditionally considered a high-risk patient population. Interestingly, the technical complexity of the implant procedure was similar in our study population, as demonstrated by the comparable fluoroscopy time as well as by the number of device deployments that were not significantly different between the two groups.

A meta-analysis by Armaganijan et al. [14] showed that elderly patients undergoing PM implantation are more prone to complications, as pneumothorax, lead dislodgements due to an increase in venous tortuosity and reduced cardiac mass for lead attachment, and cardiac perforation. On the other hand, the consistently lower rates of periprocedural complications and device related infections have supported the implantation of Micra-TPS especially in those patients at presumably higher risk of device-related complications. A major issue for concern was the risk of vascular complications in the elderly, considering the caliber of the femoral introducer and the incidence of peripheral vascular disease. Generally, a right femoral access is preferred, nevertheless in case of an unfavorable vascular anatomy, the design of the introducer and delivery system allow for a contralateral access. The absence of vascular complications in our experience encourages us to consider the leadless option in the elderly.

Likewise, feasibility and electrical performance of M-TPS in a patients population previously undergone to a transvenous lead extraction (TLE) procedure has clearly been discussed [15]. All data suggested that technical complexity of the implant procedure was similar in both patient groups, those underwent TLE and those who received M-TPS has first pacing option, in terms of fluoroscopy time and number of device deployments.
Beyond feasibility and safety of M-TPS implant in a traditionally considered "fragile" population, an important novelty is the possibility to significantly reduce the hospital stay of the patient. According to the clinical practice, patients are discharged 24-48 h after a traditional transvenous pacemaker implant. In case of leadless PM, data suggest that in appropriately selected patients, same day discharge can occur safely following M-TPS implantation [16]. Authors did not identify any difference in major complications, including problems with device function, procedural and access complications, between those discharged on the day of the procedure compared to those discharged after a traditional overnight hospital stay. In elderly patients, facilitating early mobility is pivotal as well as reducing the hospitalization length could be associated with a lower risk of bacteremia and nosocomial infections.

Moreover, M-TPS does not require a pocket formation in the sub-clavian region, when surrounding tissues are prepared without ligation. Implant in elderly patients is often affected by poor hemostasis, often related to anti-platelet or anti-coagulant concomitant therapies, with a higher risk to develop pocket hematoma. This condition is uncomfortable for the patient and increases risk of contamination and lengthening postsurgical hospital period. Nevertheless, continuing anticoagulation has the specific aim to minimize disruption of therapy and avoid unnecessary exposure to the risk of thromboembolism. Micra-TPS implant with uninterrupted anti-coagulation therapy has been seen to be satisfactorily safe [17]: despite the large-bore vascular access, the reported rate of access relates complications among patients is low. In addition, the rate of pericardial effusion does not appear to be increase.

\section{Clinical implications}

With continued ageing of the population the demand for pacing is likely to continue to increase. It is important to offer an individual, tailored approach when CIED therapy is considered in the elderly. Micra-TPS implant appears to be a safe and effective procedure even in older patients, with electrical performance and outcomes comparable with the general population at follow-up. Moreover, Micra-TPS is a valid alternative for ventricular pacing in those patients who are at high risk of infection, have no venous access or have the necessity of preserving venous access for other reason (i.e., dialysis).

\section{Conclusions}

The demand for cardiac pacing is strongly related to ageing, driving the clinical practice to look for the best solution for a considered fragile patient population. MTP-S implant is an effective and safe procedure in elderly patients, with similar electrical performance and outcome compared with younger patients at midterm follow-up.

\section{References}

1. Brignole M, Auricchio A, Baron-Esquivias G, et al. 2013 ESC Guidelines on cardiac pacing and cardiac resynchronization therapy. Eur Heart J 2013;34:2281-329. doi:10.1093/eurheartj/ eht150

2. Raatikainen MJP, Arnar DO, Merkely B, et al. A Decade of Information on the use of cardiac implantable electronic devices and interventional electrophysiological procedures in 
the European Society of Cardiology Countries: 2017 Report from the European Heart Rhythm Association. Europace 2017;19:ii1-ii90. doi: 10.1093/europace/eux258

3. Bradshaw PJ, Stobie P, Knuiman MW, at al. Trends in the incidence and prevalence of cardiac pacemaker insertions in an ageing population. Open Hear. 2014;1:1-6. doi:10.1136/ openhrt-2014-000177

4. Kusumoto FM, Schoenfeld MH, Wilkoff BL, et al. 2017 HRS expert consensus statement on cardiovascular implantable electronic device lead management and extraction. Hear Rhythm 2017;14:e503-51. doi:10.1016/j.hrthm.2017.09.001

5. Reynolds D, Duray GZ, Omar R, et al. A leadless intracardiac transcatheter pacing system. N Engl J Med 2016;374:533-41. doi:10.1056/NEJMoa1511643

6. Spickler JW, Rasor NS, Kezdi P, et al. Totally self-contained intracardiac pacemaker. J Electrocardiol 1970;3:325-31. doi:10.1016/S0022-0736(70)80059-0

7. Chew DS, Kuriachan V. Leadless cardiac pacemakers: Present and the future. Curr Opin Cardiol 2018;33:7-13. doi:10.1097/ HCO.0000000000000468

8. Chinitz L, Ritter P, Khelae SK, et al. Accelerometer-based atrioventricular synchronous pacing with a ventricular leadless pacemaker: Results from the Micra atrioventricular feasibility studies. Hear Rhythm 2018;15:1363-71. doi:10.1016/j.hrthm. 2018.05.004

9. Ammari Z, Syed M, Al-Sarie M, et al. Safety and efficacy of leadless pacemakers: a new era of pacing. J Innov Cardiac Rhythm Manag 2018;7:3215-3220. doi: 10.19102/icrm.2018. 090701

10. Ritter P, Duray GZ, Steinwender C, et al. Early performance of a miniaturized leadless cardiac pacemaker: The Micra
Transcatheter Pacing Study. Eur Heart J 2015;36(:2510-9. doi: 10.1093/eurheartj/ehv214

11. Roberts PR, Clementy N, Al Samadi F, et al. A leadless pacemaker in the real-world setting: the micra Transcatheter Pacing System Post-Approval Registry. Hear Rhythm 2017;14:13759. doi: 10.1016/j.hrthm.2017.05.017

12. El-Chami MF, Al-Samadi F, Clementy N, et al. Updated performance of the Micra transcatheter pacemaker in the realworld setting: A comparison to the investigational study and a transvenous historical control. Hear Rhythm 2018;15:1800-7. doi: 10.1016/j.hrthm.2018.08.005

13. Bongiorni MG, Della Tommasina V, Barletta V, et al. Feasibility and long-term effectiveness of a non-apical Micra pacemaker implantation in a referral centre for lead extraction. Europace 2019;21:1-7. doi: 10.1093/europace/euy116

14. Armaganijan LV, Toff WD, Nielsen JC, et al. Are elderly patients at increased risk of complications following pacemaker implantation? A meta-analysis of randomized trials. Pacing Clin Electrophysiol 2012;35131-4.

15. Zucchelli G, Barletta V, Della Tommasina V, et al. Micra pacemaker implant after cardiac implantable electronic device extraction: feasibility and long-term outcomes. Europace 2019;21:1229-36. doi: 10.1093/europace/euz160

16. Kiani S, Black GB, Rao B, et al. The safety and feasibility of same-day discharge after implantation of MICRA transcatheter leadless pacemaker system. J Atr Fibrillation 2019;12:1-5. doi: 10.4022/jafib. 2153

17. Kiani S, Black GB, Rao B, et al. Outcomes of Micra leadless pacemaker implantation with uninterrupted anticoagulation. J Cardiovasc Electrophysiol 2019;30:14313-8. doi: 10.1111/jce. 13965. 